OPEN ACCESS

Check for updates

\section{Use of sodium glucose cotransporter 2 inhibitors and risk of major cardiovascular events and heart failure: Scandinavian register based cohort study}

\author{
Björn Pasternak, ${ }^{1,2}$ Peter Ueda, ${ }^{1}$ Björn Eliasson, ${ }^{3}$ Ann-Marie Svensson, ${ }^{3,4}$ Stefan Franzén,,${ }^{4,5}$ \\ Soffia Gudbjörnsdottir, ${ }^{3,4}$ Kristian Hveem, ${ }^{6,7}$ Christian Jonasson, ${ }^{6,7}$ Viktor Wintzell, ${ }^{1}$ \\ Mads Melbye, ${ }^{2,8,9}$ Henrik Svanström ${ }^{1,2}$
}

For numbered affiliations see end of the article.

Correspondence to: B Pasternak bjorn.pasternak@ki.se

(or @Dr_Pasternak on Twitter ORCID 0000-0002-2097-8466)

Additional material is published online only. To view please visit the journal online.

Cite this as: $B M J$ 2019;366:14772 http://dx.doi.org/10.1136/bmj.l4772

Accepted: 2 July 2019

\section{ABSTRACT}

OBJECTIVE

To investigate the cardiovascular effectiveness of sodium glucose cotransporter 2 (SGLT2) inhibitors in routine clinical practice.

DESIGN

Cohort study using data from nationwide registers and an active-comparator new-user design.

SETTING

Denmark, Norway, and Sweden, from April 2013 to December 2016.

PARTICIPANTS

20983 new users of SGLT2 inhibitors and 20983 new users of dipeptidyl peptidase 4 (DPP4) inhibitors, aged 35-84, matched by age, sex, history of major cardiovascular disease, and propensity score.

\section{MAIN OUTCOME MEASURES}

Primary outcomes were major cardiovascular events (composite of myocardial infarction, stroke, and cardiovascular death) and heart failure (hospital admission for heart failure or death due to heart failure). Secondary outcomes were the individual components of the cardiovascular composite and any cause death. In the primary analyses, patients were defined as exposed from treatment start throughout follow-up (analogous to intention to treat); additional analyses were conducted with an as-treated exposure definition. Cox regression was used to estimate hazard ratios.

\section{WHAT IS ALREADY KNOWN ON THIS TOPIC}

Data from randomised cardiovascular outcome trials have shown that sodium glucose cotransporter 2 (SGLT2) inhibitors reduce the risk of major cardiovascular events and heart failure among patients with type 2 diabetes who have established cardiovascular disease or are at high cardiovascular risk The cardiovascular effectiveness of SGLT2 inhibitors in routine clinical practice is unclear

\section{WHAT THIS STUDY ADDS}

In this large cohort study based on nationwide data from three Scandinavian countries, use of SGLT2 inhibitors, as compared with use of dipeptidyl peptidase 4 (DPP4) inhibitors, was associated with a 34\% reduced risk of heart failure but was not associated with a reduced risk of major cardiovascular events Use of SGLT2 inhibitors was also associated with a $20 \%$ reduced risk of any cause death

The findings were consistent in patients with and without history of major cardiovascular disease

\section{RESULTS}

Mean age of the study cohort was 61 years, $60 \%$ were men, and $19 \%$ had a history of major cardiovascular disease. Of the total 27416 person years of follow-up in the SGLT2 inhibitor group, 22627 (83\%) was among patients who initiated dapagliflozin, 4521 (16\%) among those who initiated empagliflozin, and 268 (1\%) among those who initiated canagliflozin. During follow-up, 467 SGLT2 inhibitor users (incidence rate 17.0 events per 1000 person years) and 662 DPP4 inhibitor users (18.0) had a major cardiovascular event, whereas 130 (4.7) and 265 (7.1) had a heart failure event, respectively. Hazard ratios were 0.94 ( $95 \%$ confidence interval 0.84 to 1.06 ) for major cardiovascular events and 0.66 (0.53 to 0.81 ) for heart failure. Hazard ratios were consistent among subgroups of patients with and without history of major cardiovascular disease and with and without history of heart failure. Hazard ratios for secondary outcomes, comparing SGLT2 inhibitors with DPP4 inhibitors, were 0.99 (0.85 to 1.17) for myocardial infarction, 0.94 (0.77 to 1.15) for stroke, 0.84 (0.65 to 1.08) for cardiovascular death, and 0.80 (0.69 to 0.92) for any cause death. In the as-treated analyses, hazard ratios were 0.84 (0.72 to 0.98$)$ for major cardiovascular events, 0.55 (0.42 to 0.73) for heart failure, 0.93 (0.76 to 1.14$)$ for myocardial infarction, 0.83 (0.64 to 1.07$)$ for stroke, 0.67 (0.49 to 0.93) for cardiovascular death, and 0.75 (0.61 to 0.91$)$ for any cause death.

\section{CONCLUSIONS}

In this large Scandinavian cohort, SGLT2 inhibitor use compared with DPP4 inhibitor use was associated with reduced risk of heart failure and any cause death, but not with major cardiovascular events in the primary intention-to-treat analysis. In the additional as-treated analyses, the magnitude of the association with heart failure and any cause death became larger, and a reduced risk of major cardiovascular events that was largely driven by the cardiovascular death component was observed. These data help inform patients, practitioners, and authorities regarding the cardiovascular effectiveness of SGLT2 inhibitors in routine clinical practice.

\section{Introduction}

Sodium glucose cotransporter 2 (SGLT2) inhibitors are relatively new glucose lowering drugs used in the treatment of type 2 diabetes. Randomised placebo controlled trials of cardiovascular outcomes, conducted in patients with type 2 diabetes with a history of cardiovascular disease or who were 
otherwise at high cardiovascular risk, have shown that these drugs (including canagliflozin, dapagliflozin, and empagliflozin) reduce the risk of hard cardiovascular endpoints. ${ }^{1-3}$ A recent meta-analysis of these trials showed that SGLT2 inhibitors reduced major adverse cardiovascular events (the composite of myocardial infarction, stroke, and cardiovascular death; hazard ratio $0.89,95 \%$ confidence interval 0.83 to 0.96$)$ ), hospital admission for heart failure $(0.69,0.61$ to $0.79)$, and any cause death $(0.85,0.78$ to 0.93$)$, although heterogeneity was high for the any cause death outcome across the three trials. ${ }^{4}$

Although these data, derived from controlled trial settings, provide firm evidence for the cardiovascular efficacy of SGLT2 inhibitors among patients at high cardiovascular risk, complementary data are needed to understand to what extent they translate to cardiovascular effectiveness in the broad group of patients in routine clinical practice. A few observational studies of SGLT2 inhibitors have been published. However, these studies were focused on heart failure alone,,$^{5}$ or used designs ${ }^{6-11}$ that introduced immortal time, which has a strong potential for bias towards protective associations in favour of SGLT2 inhibitors. ${ }^{12 \text { w13 }}$ One well designed study, which investigated canagliflozin alone, reported a reduced risk of hospital admission for heart failure versus three other glucose lowering drugs, but no difference in risk of a composite of myocardial infarction or stroke (cardiovascular death was not included in this composite). ${ }^{14}$ However, that study did not have adequate data on causes of death or on total mortality, and was thus unable to investigate cardiovascular and any cause death.

In this register based cohort study, we used nationwide data from three Scandinavian countries to investigate the risk of major cardiovascular events and heart failure among new users of SGLT2 inhibitors versus an active comparator drug class, dipeptidyl peptidase 4 (DPP4) inhibitors.

\section{Methods \\ Cohort}

We conducted a cohort study, from April 2013 to December 2016, using nationwide register data from Denmark, Norway, and Sweden, and an activecomparator new-user design. ${ }^{15}$ All patients in the three countries, who were aged 35-84, and who were new users of either SGLT2 inhibitors or DPP4 inhibitors was eligible for inclusion. New use was defined as initiation of either drug class among patients with no use of either drug class within the past two years. Exclusion criteria were dialysis or renal transplantation, end stage illness, drug misuse, major pancreatic disease, no prescription drug or register entry in the national patient registers in the past year, and hospital admission for any cause in the past 30 days (definitions in web table 1 ).

\section{Data sources}

Data were obtained from registers that have nationwide coverage of each of the three study countries and linked on individual level using unique patient identifiers. The data sources are summarised in web table 2 and have been described in detail previously. ${ }^{16} 17$ Briefly, drug treatment data were obtained from the national prescription registers, which record all filled prescriptions from all pharmacies in the countries, including information on specific drug, date of drug dispensing, and amount of drug. Given their nationwide coverage, these registers permitted the identification of all patients in the three countries initiating the study drugs.

The population registers and the central bureaus of statistics provided demographic, vital status, and socioeconomic data. The national patient registers were used to obtain information on outcomes and comorbidities; these registers record all admissions to hospitals and outpatient specialist visits, and include data such as date of contact, diagnostic codes, and procedure codes. The cause of death registers, which are based on death certificates, provided cause of death data. The above data sources were used for the main analyses. For a sensitivity analysis, additional patient characteristics were obtained from the Swedish National Diabetes Register, a nationwide register to which trained physicians and nurses report clinical information regarding patients with diabetes.

\section{Confounder control and matching}

Propensity score methods were used to control for 59 potential confounder variables, including demographic and socioeconomic factors, comorbidities, drug treatments, and healthcare use (web table 3). We estimated propensity scores using logistic regression. Three variables had missing values (country of birth $(<1 \%$ missing), civil status $(<1 \%)$, and education $(3 \%))$; we handled these values by using a missing value category. ${ }^{18}$ SGLT2 inhibitor and DPP4 inhibitor users were matched, country wise, in a 1:1 ratio on propensity score using the nearest neighbour matching algorithm (caliper width 0.2 of the standard deviation of the logit score) with sex, age (five year intervals), and history of major cardiovascular disease (web table 4) as additional matching criteria. The analyses were conducted in a pooled, matched, three country cohort.

\section{Outcomes}

The coprimary outcomes were major cardiovascular events (defined as the composite of myocardial infarction, stroke, and cardiovascular death) and heart failure (defined as hospital admission for or death due to heart failure). Secondary outcomes were the components of the major cardiovascular events composite and any cause death. Cardiovascular outcomes were identified from primary diagnoses assigned during hospital admissions, captured through the national patient registers, and underlying cause of death diagnoses, captured through the cause of death registers (ICD-10 (international classification of diseases, 10th revision) codes in web table 5). The any cause death outcome was based on vital status data from the population registers. Scandinavian validation 
studies have shown that a register based strategy for identification of cardiovascular outcomes has positive predictive values of $88-100 \%$ for myocardial infarction, $69-99 \%$ for stroke, and $76-95 \%$ for heart failure (with the positive predictive values towards the higher ends of these ranges when validation assessments are based on primary diagnoses). ${ }^{19-21}$ As supplementary outcomes, we also analysed two serious adverse event outcomes of current concern with SGLT2 inhibitors: lower limb amputation and diabetic ketoacidosis (definitions in web table 5). ${ }^{21622-24}$

\section{Statistical analysis}

Patients were followed from drug initiation to outcome event, emigration, death, age 85 , or end of study. Patients were defined as exposed from treatment start throughout follow-up, analogous to an intention-totreat design. Cox proportional hazards regression, with time since start of treatment as the underlying time scale, was used to estimate hazard ratios. Absolute differences, expressed as events per 1000 person years, were calculated as hazard ratio-1 multiplied by the rate in the comparator group, with the $95 \%$ confidence interval calculated analogously. Results were considered statistically significant if the 95\% confidence interval did not overlap 1.0. Analyses were conducted using SAS software (version 9.4).

We conducted subgroup analyses of both coprimary outcomes to assess effect modification by baseline characteristics, including analyses by sex, age, history of major cardiovascular disease (acute coronary syndrome, coronary revascularisation, stroke, heart failure, or peripheral arterial disease; definitions in web table 4), and history of heart failure (web table 4). A Wald test for homogeneity was used to assess differences between subgroups, regarding $\mathrm{P}<0.05$ as consistent with significant heterogeneity. To assess consistency across data sources, we also analysed coprimary outcomes by country.

We also conducted an additional analysis with an astreated exposure definition. For this analysis, treatment duration was based on the estimated number of days covered by filled prescriptions, allowing for up to 90 days between prescriptions (gap period) and after the last prescription, which aimed to allow for irregular drug use and the capture of events occurring shortly after treatment cessation. In addition to censoring criteria applied in the primary analysis, patients were also censored at end of treatment and crossover to the other study drug. The serious adverse event outcomes were analysed with an as-treated exposure definition.

We did a preplanned sensitivity analysis of the Swedish part of the matched cohort with additional multivariable adjustment for glycated haemoglobin, blood pressure, estimated glomerular filtration rate, albuminuria, body mass index, and smoking status. Because these six variables all had some missing values (web table 6), we used multiple imputation ${ }^{25}$; using the PROC MI procedure in SAS, 10 datasets were imputed applying the Markov Chain Monte Carlo method. When assessing the results, we compared the estimates for the coprimary outcomes from this analysis with those from the primary analysis of the Swedish part of the study cohort, thus evaluating the impact of additional adjustment for the mentioned variables and hence the potential influence of these variables as unmeasured confounders in the main analysis.

We also conducted two post hoc sensitivity analyses. Firstly, we adjusted the models for country. Secondly, to test a shorter gap period in the operational definition of as-treated, we redid the analysis using 30 day and 60 day gap periods.

\section{Patient and public involvement}

No patients were involved in setting the research question, or in the design, conduct, or interpretation of the study. Being a study based on anonymised nationwide register data, there is no planned dissemination of results directly to study participants. A lay summary will be published on Karolinska Institute's website and a press release will be issued. The authors will share the results with patient advocacy and national cardiovascular groups.

\section{Results}

Cohort

During the study period, 25988 eligible new users of SGLT2 inhibitors and 94411 new users of DPP4 inhibitors were identified (fig 1; baseline characteristics before matching overall in web table 7 and by country in web tables 8-10). Following 1:1 matching by propensity score, age, sex, and history of major cardiovascular disease, the study cohort included 20983 pairs of new users of SGLT2 inhibitors and DPP4 inhibitors. The groups were well balanced on all measured covariates (table 1); mean age was 61 (standard deviation 10), 60\% were male, 19\% had history of major cardiovascular disease, and $6 \%$ had history of heart failure.

In the primary intention-to-treat analysis, duration of follow-up was a median 1.4 years (interquartile range 0.7-2.3; mean 1.5 (standard deviation 1.0)) overall, 1.1 years $(0.6-2.0 ; 1.3(0.9))$ in the SGLT2 inhibitor group, and 1.7 years $(0.8-2.7 ; 1.8(1.1))$ in the DPP4 inhibitor group. Of 27416 person years of follow-up in the SGLT2 inhibitor group, 22627 (83\%) were among patients who initiated dapagliflozin, 4521 (16\%) among those who initiated empagliflozin, and 268 (1\%) among those who initiated canagliflozin. The distribution of person years by individual DPP4 inhibitors is shown in web table 11 . The reasons for censoring according to study drug are shown in web table 12.

In the additional as-treated analysis, which was based on follow-up time from drug initiation to drug cessation or switch to the other study drug, the duration of follow-up was median 0.8 years (interquartile range 0.4-1.5; mean 1.0 (standard deviation 0.9)) overall, 0.7 years $(0.3-1.2 ; 0.9(0.7))$ among SGLT2 inhibitor users, and 0.9 years $(0.5-1.7 ; 1.2(0.9))$ among DPP4 inhibitor users. The reasons for censoring according to study drug are shown in web table 12. 


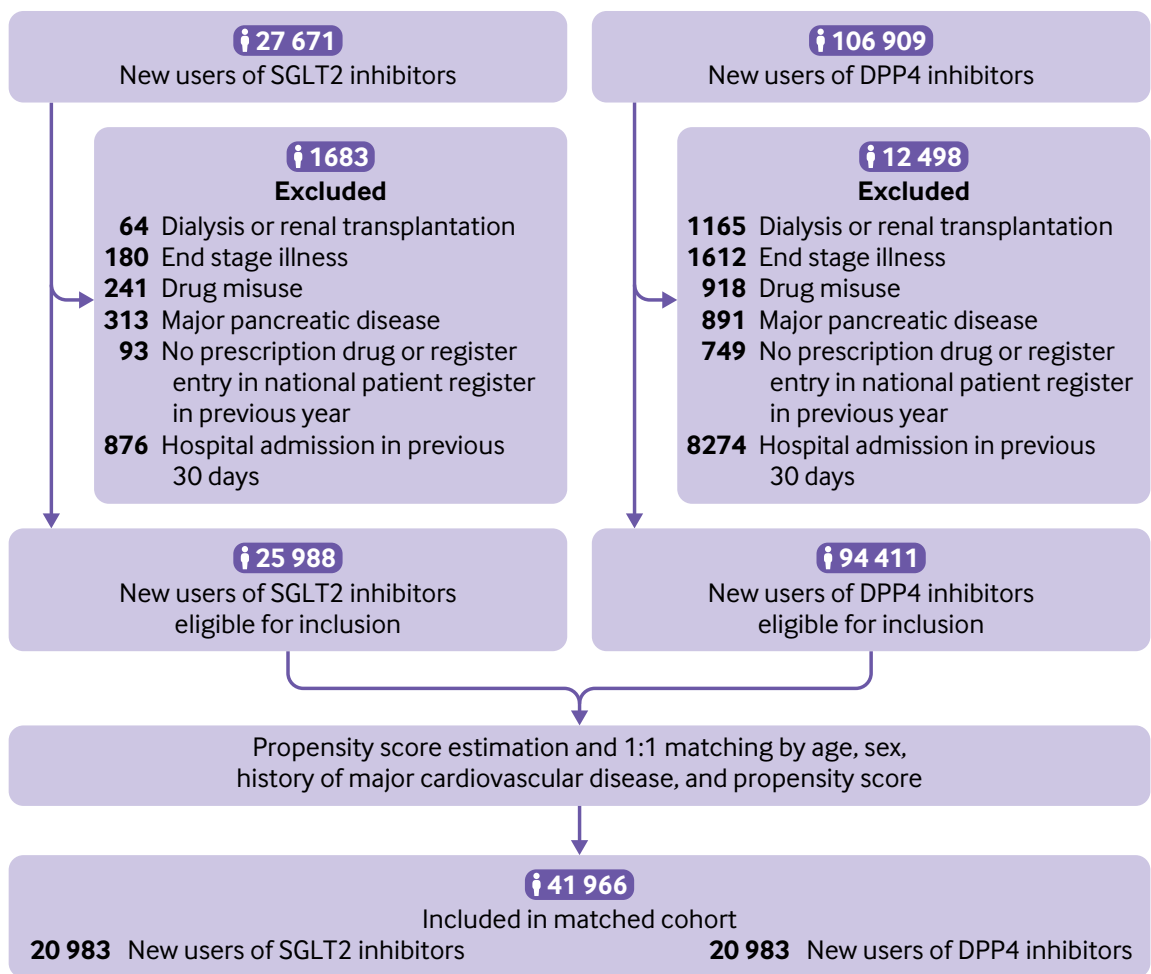

Fig 1 | Flowchart of patient inclusion in study cohort of new users of SGLT2 inhibitors and DPP4 inhibitors in Denmark, Norway, and Sweden, from April 2013 to December 2016. SGLT2=sodium-glucose cotransporter 2; DDP4=dipeptidyl peptidase 4. A single patient could be excluded because of more than one reason

Table 1 | Baseline characteristics of new users of SGLT2 inhibitors and DPP4 inhibitors matched by propensity score, age, sex, and history of major cardiovascular disease in Denmark, Norway, and Sweden, from April 2013 to December 2016. Data are number (\%) of users unless stated otherwise

\begin{tabular}{|c|c|c|}
\hline Characteristic & $\begin{array}{l}\text { SGLT2 inhibitors } \\
(\mathrm{n}=20983)\end{array}$ & $\begin{array}{l}\text { DPP4 inhibitors } \\
(\mathrm{n}=20983)\end{array}$ \\
\hline Male sex & $12589(60)$ & $12589(60)$ \\
\hline Age, mean (standard deviation) & $61(10)$ & $61(10)$ \\
\hline \multicolumn{3}{|l|}{ Country } \\
\hline Sweden & $9125(43)$ & $9125(43)$ \\
\hline Denmark & $5536(26)$ & $5536(26)$ \\
\hline Norway & $6322(30)$ & $6322(30)$ \\
\hline \multicolumn{3}{|l|}{ Place of birth } \\
\hline Scandinavia & $17609(84)$ & $17592(84)$ \\
\hline Rest of Europe & $1359(6)$ & $1389(7)$ \\
\hline Outside Europe & $1988(9)$ & $1977(9)$ \\
\hline Missing & $27(<1)$ & $25(<1)$ \\
\hline \multicolumn{3}{|l|}{ Civil status } \\
\hline Married/living with partner & $11857(57)$ & $11789(56)$ \\
\hline Single & $9086(43)$ & $9149(44)$ \\
\hline Missing & $40(<1)$ & $45(<1)$ \\
\hline \multicolumn{3}{|l|}{ Education } \\
\hline Primary school/secondary school/vocational training* & $11522(79)$ & $11519(79)$ \\
\hline Short tertiary education & $1116(8)$ & $1116(8)$ \\
\hline Medium or long tertiary education & $1704(12)$ & $1705(12)$ \\
\hline Missing & $319(2)$ & $321(2)$ \\
\hline \multicolumn{3}{|l|}{ Year of cohort entryt } \\
\hline 2013 & $1278(6)$ & $3872(18)$ \\
\hline 2014 & $4364(21)$ & $5292(25)$ \\
\hline 2015 & $6081(29)$ & $5884(28)$ \\
\hline 2016 & $9260(44)$ & $5935(28)$ \\
\hline \multicolumn{3}{|l|}{ Comorbidities } \\
\hline Acute coronary syndrome & $1543(7)$ & $1578(8)$ \\
\hline Other ischaemic heart disease & $3648(17)$ & $3662(17)$ \\
\hline Heart failure/cardiomyopathy & $1164(6)$ & $1174(6)$ \\
\hline
\end{tabular}

\section{Primary and secondary outcomes}

Figure 2 shows the cumulative incidences of coprimary and secondary outcomes. During follow-up, 467 SGLT2 inhibitor users (incidence rate 17.0 events per 1000 person years) and 662 DPP4 inhibitor users (18.0) had a major cardiovascular event, whereas 130 (4.7) and 265 (7.1) users had a heart failure event, respectively. The hazard ratios, comparing SGLT2 inhibitors with DPP4 inhibitors, were 0.94 (95\% confidence interval 0.84 to 1.06 ) for major cardiovascular events and 0.66 (0.53 to 0.81) for heart failure (table 2).

Hazard ratios for secondary outcomes were 0.99 (95\% confidence interval 0.85 to 1.17 ) for myocardial infarction, 0.94 (0.77 to 1.15$)$ for stroke, 0.84 (0.65 to 1.08) for cardiovascular death, and 0.80 (0.69 to 0.92) for any cause death (table 2). In the additional as-treated analysis, the hazard ratio for the major cardiovascular events composite outcome was 0.84 (0.72 to 0.98 ), which appeared to be primarily driven by the cardiovascular death component (table 2). The hazard ratio for heart failure was 0.55 (0.42 to 0.73 ; table 2).

\section{Subgroup analyses}

Subgroup analyses are shown in figure 3. For both the outcome of major cardiovascular events and the outcome of heart failure, we saw substantial differences in incidence rates across subgroups, with the highest rates of outcome events observed among patients with history of major cardiovascular disease and those with history of heart failure. For the 


\begin{tabular}{|c|c|c|}
\hline \multicolumn{3}{|l|}{ Table 1 | Continued } \\
\hline Characteristic & $\begin{array}{l}\text { SGLT2 inhibitors } \\
(\mathrm{n}=20983)\end{array}$ & $\begin{array}{l}\text { DPP4 inhibitors } \\
(\mathrm{n}=20983)\end{array}$ \\
\hline Valve disorders & $473(2)$ & $477(2)$ \\
\hline Stroke & $766(4)$ & $745(4)$ \\
\hline Other cerebrovascular disease & $873(4)$ & $854(4)$ \\
\hline Atrial fibrillation & $1439(7)$ & $1408(7)$ \\
\hline Other arrhythmia & $916(4)$ & $833(4)$ \\
\hline Coronary revascularisation in past year & $284(1)$ & $282(1)$ \\
\hline \multicolumn{3}{|l|}{ Other cardiac surgery or invasive procedure in past } \\
\hline year & $127(1)$ & $104(<1)$ \\
\hline Chronic obstructive pulmonary disease & $795(4)$ & $759(4)$ \\
\hline Other lung disease & $1448(7)$ & $1470(7)$ \\
\hline Venous thromboembolism & $471(2)$ & $447(2)$ \\
\hline Cancer & $1380(7)$ & $1406(7)$ \\
\hline Liver disease & $425(2)$ & $434(2)$ \\
\hline Rheumatic disease & $626(3)$ & $600(3)$ \\
\hline Psychiatric disorder & $2091(10)$ & $2120(10)$ \\
\hline Fracture in the past year & $346(2)$ & $344(2)$ \\
\hline Arterial disease (including amputation) & $1331(6)$ & $1273(6)$ \\
\hline Renal disease & $955(5)$ & $909(4)$ \\
\hline Diabetic complications & $6169(29)$ & $6172(29)$ \\
\hline \multicolumn{3}{|l|}{ Hospital admissions and outpatient visits in the past year } \\
\hline Hospital admissions due to cardiovascular causes & $881(4)$ & $841(4)$ \\
\hline Hospital admissions due to type 2 diabetes & $184(1)$ & $180(1)$ \\
\hline Hospital admissions due to other causes & $2565(12)$ & $2559(12)$ \\
\hline Outpatient visits due to cardiovascular causes & $2056(10)$ & $1975(9)$ \\
\hline Outpatient visits due to type 2 diabetes & $4659(22)$ & $4657(22)$ \\
\hline Outpatient visits due to other causes & $11780(56)$ & $11655(56)$ \\
\hline \multicolumn{3}{|l|}{ Use of diabetes drugs in past six months } \\
\hline Metformin & $16540(79)$ & $16659(79)$ \\
\hline Sulphonylureas & $4386(21)$ & $4376(21)$ \\
\hline Insulin & $6636(32)$ & $6712(32)$ \\
\hline GLP1 receptor agonists & $2143(10)$ & $2112(10)$ \\
\hline Other diabetes drugs (glitazones, glinides, acarbose) & $654(3)$ & $649(3)$ \\
\hline No diabetes drug & $1685(8)$ & $1672(8)$ \\
\hline \multicolumn{3}{|l|}{ Time since use of first diabetes drug (years) } \\
\hline$<1$ & $2542(12)$ & $2532(12)$ \\
\hline $1-2$ & $2496(12)$ & $2559(12)$ \\
\hline $3-4$ & $2528(12)$ & $2567(12)$ \\
\hline $5-6$ & 2649 (13) & $2578(12)$ \\
\hline$\geq 7$ & $10768(51)$ & $10747(51)$ \\
\hline \multicolumn{3}{|l|}{ Use other drugs in past year } \\
\hline ARB/ACE-I & $13924(66)$ & $13905(66)$ \\
\hline Calcium channel blocker & $6250(30)$ & $6242(30)$ \\
\hline Loop diuretic* & $2114(14)$ & $2106(14)$ \\
\hline Other diuretic* & $2592(18)$ & $2591(18)$ \\
\hline$\beta$ blocker & $7453(36)$ & $7411(35)$ \\
\hline Digoxin & $397(2)$ & $363(2)$ \\
\hline Nitrate & $1516(7)$ & $1487(7)$ \\
\hline Platelet inhibitors & $7643(36)$ & $7624(36)$ \\
\hline Anticoagulant & $1454(7)$ & $1393(7)$ \\
\hline Lipid lowering drug & $14145(67)$ & $14045(67)$ \\
\hline Antidepressant & $3261(16)$ & $3280(16)$ \\
\hline Antipsychotic & $772(4)$ & $794(4)$ \\
\hline Anxiolytic, hypnotic, or sedative & $3757(18)$ & $3727(18)$ \\
\hline$\beta 2$ agonist inhalant & $2009(10)$ & $1951(9)$ \\
\hline Anticholinergic inhalant & $632(3)$ & $592(3)$ \\
\hline Glucocorticoid inhalant & $2079(10)$ & $2021(10)$ \\
\hline Oral glucocorticoid & $1522(7)$ & $1520(7)$ \\
\hline NSAID & $5415(26)$ & $5265(25)$ \\
\hline Opioid & $4098(20)$ & 4038 (19) \\
\hline \multicolumn{3}{|l|}{ No of drugs used in past year* } \\
\hline $0-5$ & 2842 (19) & $2929(20)$ \\
\hline $6-10$ & 5939 (41) & $5937(40)$ \\
\hline 11-15 & $3669(25)$ & $3596(25)$ \\
\hline$\geq 16$ & $2211(15)$ & 2199 (15) \\
\hline
\end{tabular}

$\mathrm{ACE}-\mathrm{I}=$ angiotensin converting enzyme inhibitor; $\mathrm{ARB}=$ angiotensin receptor blocker; $\mathrm{DDP} 4=$ dipeptidyl peptidase 4 ; NSAID=non-steroidal anti-inflammatory drug; SGLT2=sodium-glucose cotransporter 2; GLP1=glucagon-like peptide 1 *Variable available in Denmark and Sweden but not Norway. Data reported for Denmark and Sweden only.

tYear of cohort entry was not included in the propensity score. outcome of major cardiovascular events, we saw no significant heterogeneity in analyses of the association with SGLT2 inhibitors according to history of major cardiovascular disease and history of heart failure. However, we saw significant heterogeneity by sex and age. For the outcome of heart failure, we saw no significant heterogeneity across subgroups. Results by country are shown in web table 13.

\section{Sensitivity analyses}

The preplanned sensitivity analysis based on the Swedish part of the cohort was-in addition to confounding control through matching by propensity score, age, sex, and history of major cardiovascular disease-also adjusted for glycated haemoglobin, blood pressure, glomerular filtration rate, albuminuria, body mass index, and smoking status. Web table 6 shows the distribution of these variables and web table 14 shows the estimates from the multivariable model with multiple imputation. Comparing SGLT2 inhibitors with DPP4 inhibitors, hazard ratios for major cardiovascular events (1.04 (95\% confidence interval 0.87 to 1.24$)$ ) and heart failure (0.83 (0.61 to 1.12)) in these additionally adjusted analyses were similar to those observed in the Swedish part of the matched cohort without such adjustment (1.01 (0.85 to 1.21) and 0.77 (0.57 to 1.04), respectively).

In post hoc sensitivity analyses (web table 15), hazard ratios of the coprimary outcomes with additional adjustment for country were unchanged compared with the primary analyses. Hazard ratios of the coprimary outcomes of the as-treated analysis with the gap period reduced to 30 days and 60 days, respectively, were similar to those of the main astreated analysis.

\section{Supplementary serious adverse event outcomes}

The incidence rate of lower limb amputation was 3.1 events per 1000 person years among SGLT2 inhibitor users and 2.6 events per 1000 person years among DPP4 inhibitor users, whereas the rates of diabetic ketoacidosis were 1.4 and 0.6 , respectively. Hazard ratios comparing SGLT2 inhibitors with DPP4 inhibitors were 1.26 (95\% confidence interval 0.88 to 1.81 ) for lower limb amputation and 2.14 (1.17 to 4.09) for diabetic ketoacidosis (web table 16).

\section{Discussion \\ Main findings}

This large Scandinavian cohort study investigated the cardiovascular effectiveness of SGLT2 inhibitors in routine clinical practice. Use of SGLT2 inhibitors, compared with use of DPP4 inhibitors, was not associated with a reduced risk of the coprimary outcome major cardiovascular events or any of the components of this composite outcome (the secondary outcomes myocardial infarction, stroke, and cardiovascular death). By contrast, use of SGLT2 inhibitors was associated with a 34\% reduced risk of the coprimary outcome heart failure and a $20 \%$ reduced risk of the secondary outcome any cause 


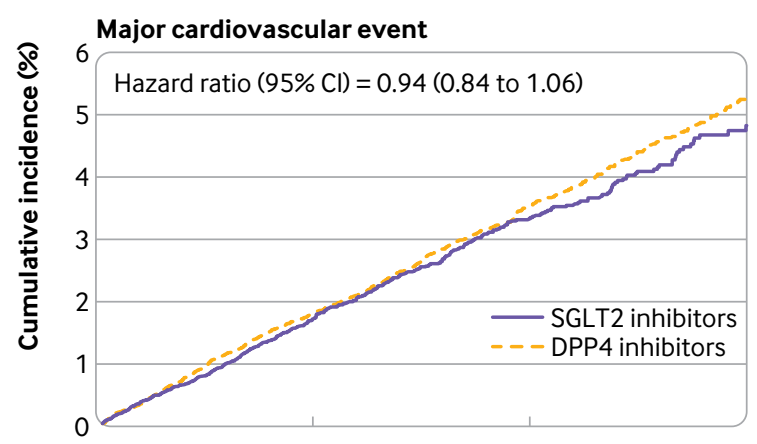

No at risk

SGLT2 inhibitors

$$
20983
$$

11407

DPP4 inhibitors 20983
5350

8660
1206

3553

\section{Heart failure}

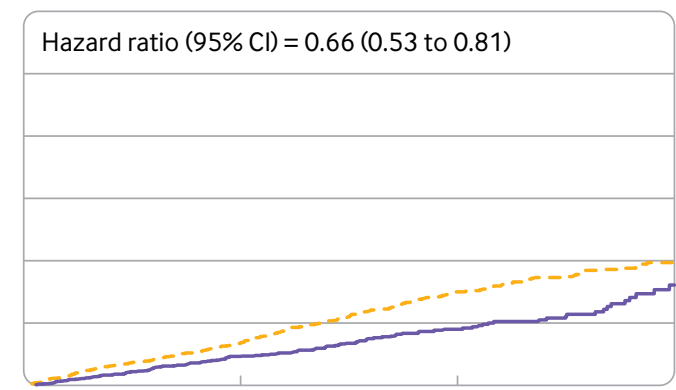

20983

11524

5449

1226

20983

14738

8773

3638
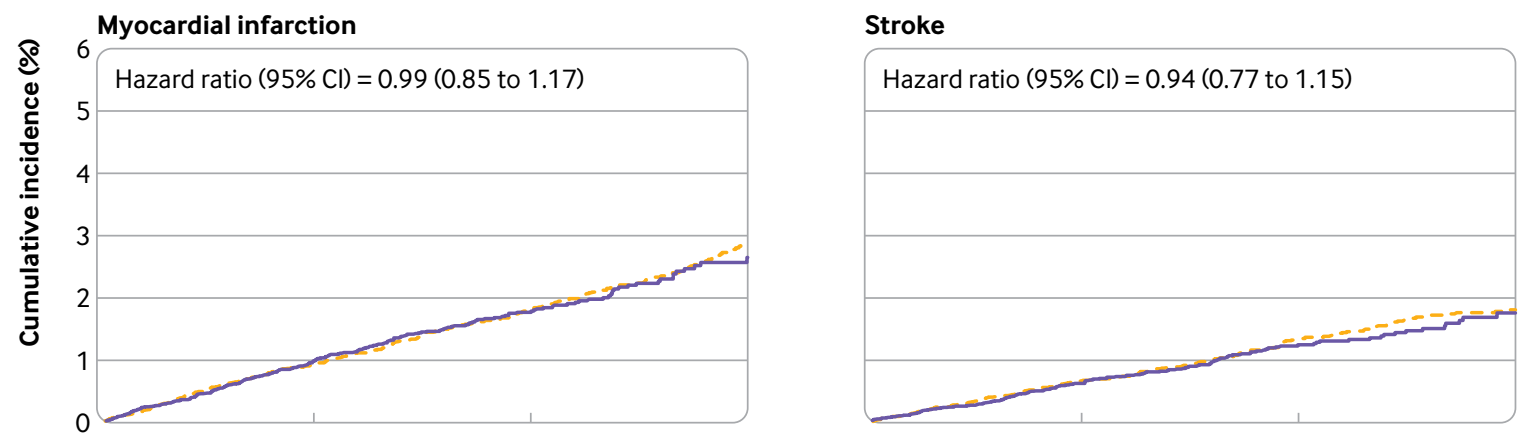

No at risk

SGLT2 inhibitors 20983 11470 5411 1222 20983

11505

5430

DPP4 inhibitors 20983

14708

8751

3610

20983

14739

8787

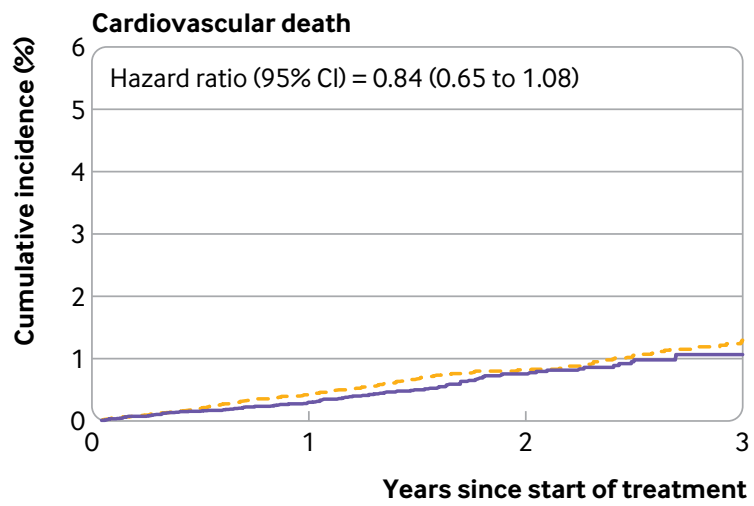

No at risk

SGLT2 inhibitors

$$
20983
$$

DPP4 inhibitors

20983

14824

5494

1243

20983

11571

5494

Any cause death

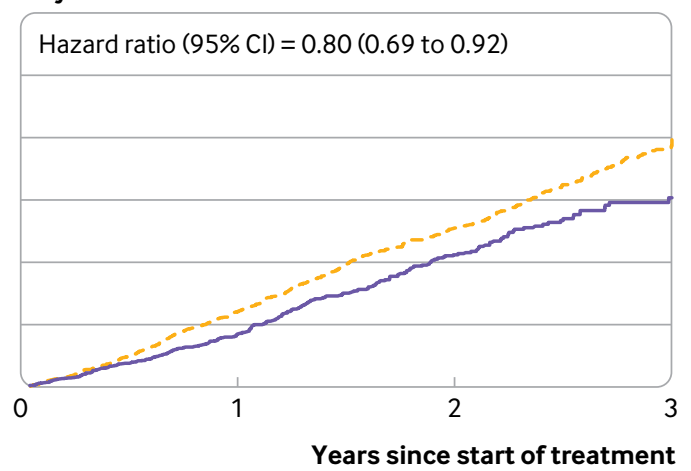

Fig 2 | Cumulative incidence of cardiovascular events associated with use of SGLT2 inhibitors, compared with use of DPP4 inhibitors-primary analyses (intention-to-treat exposure definition). Owing to declining numbers of patients at risk and outcome events, cumulative incidence curves were truncated at three years (maximum follow-up in the study was three years and nine months)

death. Nineteen per cent of patients in the cohort had history of major cardiovascular disease; hazard ratios for the coprimary outcomes were consistent among subgroups of patients with and without such history.
Interpretation and comparison with previous studies A recent meta-analysis of the three randomised cardiovascular outcome trials published so far confirmed the benefit of SGLT2 inhibitors on hospital 


\begin{tabular}{|c|c|c|c|c|c|c|}
\hline & \multicolumn{2}{|c|}{ SGLT2 inhibitors $(n=20983)$} & \multicolumn{2}{|c|}{ DPP4 inhibitors ( $n=20983)$} & \multirow[b]{2}{*}{$\begin{array}{l}\text { Hazard ratio } \\
(95 \% \mathrm{Cl})\end{array}$} & \multirow{2}{*}{$\begin{array}{l}\text { Absolute difference } \\
\text { (No of events per } \\
1000 \text { person years; } \\
95 \% \mathrm{Cl} \text { ) }\end{array}$} \\
\hline & $\begin{array}{l}\text { No of } \\
\text { events }\end{array}$ & $\begin{array}{l}\text { Incidence rate } \\
\text { (No of events per } \\
1000 \text { person years) }\end{array}$ & $\begin{array}{l}\text { No of } \\
\text { events }\end{array}$ & $\begin{array}{l}\text { Incidence rate } \\
\text { (No of events per } \\
1000 \text { person years) }\end{array}$ & & \\
\hline \multicolumn{7}{|c|}{ Primary analyses (intention-to-treat exposure definition) } \\
\hline \multicolumn{7}{|c|}{ Coprimary outcomes } \\
\hline Major cardiovascular events* & 467 & 17.0 & 662 & 18.0 & $0.94(0.84$ to 1.06$)$ & $-1.1(-2.9$ to 1.1$)$ \\
\hline Heart failuret & 130 & 4.7 & 265 & 7.1 & $0.66(0.53$ to 0.81$)$ & $-2.4(-3.3$ to -1.3$)$ \\
\hline \multicolumn{7}{|l|}{ Secondary outcomes } \\
\hline Myocardial infarction & 259 & 9.4 & 349 & 9.4 & 0.99 (0.85 to 1.17$)$ & $-0.1(-1.4$ to 1.6$)$ \\
\hline Stroke & 169 & 6.1 & 238 & 6.4 & $0.94(0.77$ to 1.15$)$ & $-0.4(-1.5$ to 1.0$)$ \\
\hline Cardiovascular death & 100 & 3.6 & 163 & 4.4 & $0.84(0.65$ to 1.08$)$ & $-0.7(-1.5$ to 0.4$)$ \\
\hline Any cause death & 282 & 10.2 & 494 & 13.2 & $0.80(0.69$ to 0.92$)$ & $-2.6(-4.1$ to -1.1$)$ \\
\hline \multicolumn{7}{|c|}{ Additional analyses (as-treated exposure definition) } \\
\hline \multicolumn{7}{|l|}{ Coprimary outcomes } \\
\hline Major cardiovascular events* & 281 & 15.0 & 433 & 17.5 & $0.84(0.72$ to 0.98$)$ & $-2.8(-4.9$ to -0.4$)$ \\
\hline Heart failuret & 73 & 3.9 & 172 & 6.9 & $0.55(0.42$ to 0.73$)$ & $-3.1(-4.0$ to -1.9$)$ \\
\hline \multicolumn{7}{|l|}{ Secondary outcomes } \\
\hline Myocardial infarction & 163 & 8.7 & 227 & 9.1 & 0.93 (0.76 to 1.14) & $-0.6(-2.2$ to 1.3$)$ \\
\hline Stroke & 98 & 5.2 & 153 & 6.1 & 0.83 (0.64 to 1.07$)$ & $-1.0(-2.2$ to 0.3$)$ \\
\hline Cardiovascular death & 58 & 3.1 & 113 & 4.5 & $0.67(0.49$ to 0.93$)$ & $-1.5(-2.3$ to -0.3$)$ \\
\hline Any cause death & 155 & 8.2 & 277 & 11.1 & $0.75(0.61$ to 0.91$)$ & $-2.8(-4.3$ to -1.0$)$ \\
\hline
\end{tabular}

admission for heart failure (hazard ratio 0.69 (95\% confidence interval 0.61 to 0.79)); an effect that was similar in patients with $(0.71(0.62$ to 0.82$))$ and without (0.64 (0.48 to 0.85$)$ ) atherosclerotic cardiovascular disease at baseline. ${ }^{4}$ In accordance with these findings, use of SGLT2 inhibitors in our study was associated with a risk reduction of heart failure that was of similar magnitude and consistent in subgroups of patients with and without major cardiovascular disease as well as those with and without pre-existing heart failure. This finding supports the notion that SGLT2 inhibitors could reduce risk of heart failure outcomes in a broad group of patients at varying levels of cardiovascular risk. However, in the light of the large differences in heart failure event rates between patients with and without history of heart failure as well as with and without history of cardiovascular disease, but with similar hazard ratios, patients with such histories are likely to derive the largest absolute benefit.

By contrast to our finding of no association between SGLT2 inhibitors and major cardiovascular events in the primary analysis with an intention-to-treat exposure definition, the meta-analysis ${ }^{4}$ showed that SGLT2 inhibitors reduced risk of major adverse cardiovascular events (the composite of myocardial infarction, stroke, and cardiovascular death; hazard ratio 0.89 (95\% confidence interval 0.83 to 0.96$)$ ). The protective effect seemed to be confined to patients with established atherosclerotic cardiovascular disease $(0.86$ ( 0.80 to 0.93)) whereas patients without such disease (but who had multiple cardiovascular risk factors, according to the design of the included trials) did not benefit (1.00 (0.87 to 1.16$)$ ). In our study, $81 \%$ of the study population did not have history of major cardiovascular disease at baseline, although subgroup analyses of those with such history also showed no association between use of SGLT2 inhibitors and risk of major cardiovascular events. Importantly, $83 \%$ of the person years of follow-up in our study were among patients who initiated dapagliflozin; in the DECLARE-TIMI 58 trial of dapagliflozin, researchers saw no reduction of major adverse cardiovascular events (0.93 (0.84 to 1.03)), not even in patients with established cardiovascular disease (although the hazard ratio in that subgroup tended towards possible benefit; 0.90 (0.79 to 1.02$))^{1}$

A few cohort studies have assessed cardiovascular risks associated with SGLT2 inhibitors. A multinational database study (CVD-REAL) ${ }^{7-10}$ and a study based on US Military Health System data (EASEL) ${ }^{11}$ reported reduced risk of cardiovascular events and death associated with SGLT2 inhibitors but had study design limitations. An important requirement for entry in a new user, pharmacoepidemiological cohort is that individuals are free of both the study drug and comparator drug during a washout period before entry. ${ }^{15}$ At the cohort creation step in CVD-REAL and EASEL, the assessment of new user status of SGLT2 inhibitor users and comparator drug users was separated, only taking into consideration previous use within the respective drug group. Further, drug exposure group assignment followed a hierarchical structure, giving preference to the SGLT2 inhibitor group. Hence, as outlined elsewhere, ${ }^{12} 13$ immortal time was introduced in patients initiating an SGLT2 inhibitor after having used a comparator drug, because these patients will have survived until the date of SGLT2 inhibitor initiation whereas the time on comparator drug was not included in the analysis. This immortal time would bias results in a protective direction favouring SGLT2 inhibitors. This bias likely explains why the hazard ratios for any cause death in those studies were around 0.50, whereas in our study, 


\begin{tabular}{|c|c|c|c|c|c|c|c|c|}
\hline & \multicolumn{2}{|c|}{ SGLT2 inhibitors } & \multicolumn{2}{|c|}{ DPP4 inhibitors } & \multirow[b]{2}{*}{$\begin{array}{c}\text { Hazard ratio } \\
(95 \% \mathrm{Cl})\end{array}$} & \multirow[b]{2}{*}{$\begin{array}{c}\text { Hazard ratio } \\
(95 \% \mathrm{Cl})\end{array}$} & \multirow[b]{2}{*}{$\begin{array}{c}P \\
\text { value }\end{array}$} & \multirow[b]{2}{*}{$\begin{array}{c}\text { Absolute difference, } \\
\text { events }(95 \% \mathrm{Cl}) \text { per } \\
1000 \text { person years }\end{array}$} \\
\hline & Patients & $\begin{array}{l}\text { Events/events } \\
\text { per } 1000 \\
\text { person years }\end{array}$ & Patients & $\begin{array}{c}\text { Events/events } \\
\text { per } 1000 \\
\text { person years }\end{array}$ & & & & \\
\hline \multicolumn{9}{|c|}{ Major cardiovascular events } \\
\hline Primary analysis & 20983 & $467 / 17.0$ & 20983 & $662 / 18.0$ & $0.94(0.84$ to 1.06$)$ & $\rightarrow$ & & $-1.1(-2.9$ to 1.1$)$ \\
\hline \multicolumn{9}{|l|}{ Sex } \\
\hline Female & 8394 & $115 / 10.1$ & 8394 & $193 / 12.9$ & $0.76(0.61$ to 0.96$)$ & \multirow{2}{*}{$\rightarrow$} & \multirow{2}{*}{0.03} & $-3.1(-5.0$ to -0.5$)$ \\
\hline Male & 12589 & $352 / 21.9$ & 12589 & $469 / 21.4$ & 1.03 (0.89 to 1.18$)$ & & & $0.6(-2.4$ to 3.9$)$ \\
\hline \multicolumn{9}{|l|}{ Age } \\
\hline$<65$ & 12773 & $224 / 13.1$ & 12773 & $268 / 11.6$ & $1.13(0.94$ to 1.35$)$ & \multirow[b]{2}{*}{$\rightarrow$} & \multirow{2}{*}{0.01} & $1.5(-0.7$ to 4.0$)$ \\
\hline$\geq 65$ & 8210 & $243 / 23.5$ & 8210 & $394 / 29.0$ & $0.81(0.69$ to 0.96$)$ & & & $-5.5(-9.0$ to -1.2$)$ \\
\hline \multicolumn{9}{|c|}{ History of major cardiovascular disease } \\
\hline No & 16962 & $269 / 11.9$ & 16962 & $397 / 13.3$ & $0.90(0.77$ to 1.05$)$ & - & \multirow{2}{*}{0.22} & $-1.3(-3.0$ to 0.7$)$ \\
\hline Yes & 4021 & $198 / 40.7$ & 4021 & $265 / 38.8$ & 1.05 (0.87 to 1.26$)$ & & & $1.9(-5.0$ to 10.1$)$ \\
\hline \multicolumn{9}{|c|}{ History of heart failure } \\
\hline No & 19819 & $389 / 14.9$ & 19809 & $556 / 16.0$ & 0.94 (0.82 to 1.07$)$ & & \multirow{2}{*}{0.49} & $-1.0(-2.9$ to 1.1$)$ \\
\hline Yes & 1164 & $78 / 58.4$ & 1174 & $106 / 54.6$ & 1.05 (0.78 to 1.41$)$ & & & $2.7(-12.0$ to 22.4$)$ \\
\hline \multicolumn{9}{|l|}{ Heart failure } \\
\hline Primary analysis & 20983 & $130 / 4.7$ & 20983 & $265 / 7.1$ & $0.66(0.53$ to 0.81$)$ & $\rightarrow$ & & $-2.4(-3.3$ to -1.3$)$ \\
\hline \multicolumn{9}{|l|}{ Sex } \\
\hline Female & 8394 & $47 / 4.1$ & 8394 & $80 / 5.3$ & 0.78 (0.54 to 1.12$)$ & \multirow[b]{2}{*}{$\longrightarrow$} & \multirow{2}{*}{0.29} & $-1.2(-2.5$ to 0.6$)$ \\
\hline Male & 12589 & $83 / 5.1$ & 12589 & $185 / 8.4$ & 0.61 (0.47 to 0.79 ) & & & $-3.3(-4.4$ to -1.8$)$ \\
\hline \multicolumn{9}{|l|}{ Age } \\
\hline$<65$ & 12773 & $55 / 3.2$ & 12773 & $94 / 4.0$ & $0.81(0.58$ to 1.13$)$ & \multirow[b]{2}{*}{$\longrightarrow$} & \multirow{2}{*}{0.13} & $-0.8(-1.7$ to 0.5$)$ \\
\hline$\geq 65$ & 8210 & $75 / 7.2$ & 8210 & $171 / 12.4$ & $0.58(0.44$ to 0.76$)$ & & & $-5.2(-7.0$ to -3.0$)$ \\
\hline \multicolumn{9}{|c|}{ History of major cardiovascular disease } \\
\hline No & 16962 & $50 / 2.2$ & 16962 & $95 / 3.1$ & 0.72 (0.51 to 1.02$)$ & $\longrightarrow$ & \multirow{2}{*}{0.63} & $-0.9(-1.5$ to 0.1$)$ \\
\hline Yes & 4021 & $80 / 16.3$ & 4021 & $170 / 24.7$ & 0.65 (0.50 to 0.85$)$ & $\longrightarrow$ & & $-8.6(-12.4$ to -3.7$)$ \\
\hline \multicolumn{9}{|c|}{ History of heart failure } \\
\hline No & 19819 & $67 / 2.5$ & 19809 & $137 / 3.9$ & $0.68(0.51$ to 0.91$)$ & $\longrightarrow$ & 0.5 & $-1.2(-1.9$ to -0.3$)$ \\
\hline Yes & 1164 & $63 / 47.8$ & 1174 & $128 / 68.3$ & $0.69(0.51$ to 0.93$)$ & $\longrightarrow$ & 0.95 & $-21.2(-33.5$ to -4.8$)$ \\
\hline & & & & & 0. & 0.5 & 2 & \\
\hline
\end{tabular}

Fig 3 | Subgroup analyses of association between use of SGLT2 inhibitors and use of DPP4 inhibitors and risk of major cardiovascular events and heart failure. SGLT2=sodium-glucose cotransporter 2; DDP4=dipeptidyl peptidase 4

which excluded users of either study drug before cohort entry, the hazard ratio was 0.80 . A third study, based on US claims, Medicare, and Medicaid data and focused on heart failure alone, similarly separated the inclusion SGLT2 inhibitors and comparator drugs. ${ }^{6}$

In another study based on Korean data investigating heart failure in SGLT2 inhibitor users versus DPP4 inhibitor users, the hazard ratio for heart failure was similar to that in our study. ${ }^{5}$ A well designed study based on US claims data compared canagliflozin with three individual glucose lowering drug classes, including DPP4 inhibitors. ${ }^{14}$ In accordance with our study, it reported a reduced risk of heart failure that was of similar magnitude in both patients with and without history of heart failure, whereas no reduced risk of cardiovascular events was observed. That study, however, had neither information on causes of death, which precluded the inclusion of cardiovascular death in the cardiovascular composite, nor adequate data on total mortality. Similarly, another well designed study used claims data to compare empagliflozin with sitagliptin; focused on the outcome of heart failure, it found a hazard ratio of similar magnitude as that in our study. ${ }^{26}$

Our study expands on previous observational data. Building on nationwide populations from three countries and adequately implementing an active-comparator new-user design, it provides the full spectrum of relevant outcomes including cardiovascular and any cause death for the SGLT2 inhibitor class, mainly based on dapagliflozin. Further, the results are supported by the consistency of the estimates when taking glycated haemoglobin, blood pressure, glomerular filtration rate, albuminuria, body mass index, and smoking into account in a sensitivity analysis based on an entire country.

We conducted our main analyses using an intentionto-treat exposure definition. Hence, when interpreting the estimates, it is important to recognise that the analyses explicitly aimed to include all available person time among patients initiating SGLT2 inhibitors, regardless of subsequent downstream 
events, for instance, treatment cessation or switch. The main analyses thus investigated the overall clinical cardiovascular impact of initiating SGLT2 inhibitors. In the additional analysis with an as-treated definition of exposure, the magnitude of the protective association between SGLT2 inhibitors and both heart failure and any cause death became larger and a reduced risk of major cardiovascular events that was largely driven by the cardiovascular death component was observed. This finding indicates that the cardiovascular impact of SGLT2 inhibitors could be stronger during the time patients stay on the drug.

Our analysis of diabetic ketoacidosis, showing a twofold increased risk, is consistent with our previous study in which the comparator group was glucagonlike peptide 1 receptor agonists and that was based on nationwide data from Denmark and Sweden. ${ }^{16}$ The current study expands on those findings by using DPP4 inhibitors as a comparator and by including data from an additional country (Norway). The results are in line with a relatively consistent body of literature supporting an increased risk of diabetic ketoacidosis with SGLT2 inhibitors. ${ }^{116222327}$ Further, the estimate for lower limb amputation in our study was inconclusive (hazard ratio 1.26 (95\% confidence interval 0.88 to 1.81 )), adding to the uncertainty regarding this potential adverse event, with some data supporting an association ${ }^{2162428}$ and other data not in support. $^{132829}$

\section{Limitations}

Although we took meticulous care to control for confounding, the observational design of the study and the absence of randomisation means that residual confounding cannot be ruled out. However, if confounding were present, it would have to selectively bias the association in a protective direction for SGLT2 inhibitors for one cardiovascular outcome (heart failure) but not others (eg, myocardial infarction and stroke).

The study period was the first four years following the approval of SGLT2 inhibitors for clinical use. Although $25 \%$ of the SGLT2 inhibitor group were followed up for two years and more (as indicated by the upper limit of the interquartile range), median follow-up among SGLT2 inhibitor users was 1.1 years. A longer duration of follow-up might be required to detect differences in the major cardiovascular events outcome.

With patients who initiated dapagliflozin contributing with the majority of follow-up time in our study, the results mainly apply to this specific drug. Investigation of cardiovascular events associated with different individual SGLT2 inhibitors and their headto-head effectiveness represent important topics for future studies.

We chose DPP4 inhibitors as the active comparator because these drugs are also one of the newer glucose lowering drug classes, used as second line agents. We aimed to investigate the clinical effectiveness of SGLT2 inhibitors, which relies on the comparator group being risk neutral. With regard to the outcome of major cardiovascular events, all four published cardiovascular outcome trials of DPP4 inhibitors (saxagliptin, sitagliptin, alogliptin, and linagliptin) were neutral. ${ }^{30-33}$ With regard to heart failure, three of the trials were neutral and one, investigating saxagliptin, reported a small increase in risk, ${ }^{30-33}$ whereas large observational studies found no increased risk associated with DPP4 inhibitors or with saxagliptin specifically. ${ }^{34} 35$ In our study, saxagliptin represented only $4 \%$ of DPP4 inhibitor exposure. Even if DPP4 inhibitors were not risk neutral, the analysis would still reflect the head-to-head comparative effectiveness of SGLT2 inhibitors versus DPP4 inhibitors.

\section{Conclusion}

In this large Scandinavian cohort, use of SGLT2 inhibitors, as compared with DPP4 inhibitors, was associated with reduced risk of heart failure and any cause death but not with major cardiovascular events in the primary intention-to-treat analysis. In the additional as-treated analyses, the magnitude of the association with heart failure and any cause death became larger, and a reduced risk of major cardiovascular events that was largely driven by the cardiovascular death component was observed. These data help inform patients, practitioners, and authorities regarding the cardiovascular effectiveness of SGLT2 inhibitors in routine clinical practice.

\section{AUTHOR AFFILIATIONS}

${ }^{1}$ Clinical Epidemiology Division, Department of Medicine Solna, Karolinska Institutet, 17176 Stockholm, Sweden

${ }^{2}$ Department of Epidemiology Research, Statens Serum Institut, Copenhagen, Denmark

${ }^{3}$ Department of Molecular and Clinical Medicine, Institute of Medicine, University of Gothenburg, Gothenburg, Sweden

${ }^{4}$ Swedish National Diabetes Register, Västra Götalandsregionen, Gothenburg, Sweden

${ }^{5}$ Health Metrics, Department of Public Health and Community Medicine, Sahlgrenska Academy, University of Gothenburg, Gothenburg, Sweden

${ }^{6} \mathrm{~K}$ G Jebsen Centre for Genetic Epidemiology, Department of Public Health and Nursing, Faculty of Medicine and Health Science, Norwegian University of Science and Technology, Trondheim, Norway ${ }^{7}$ HUNT Research Centre, Faculty of Medicine, Norwegian University of Science and Technology, Levanger, Norway

${ }^{8}$ Department of Clinical Medicine, University of Copenhagen, Copenhagen, Denmark

${ }^{9}$ Department of Medicine, Stanford University School of Medicine, Stanford, CA, USA

Data from the Norwegian Patient Registry have been used in this publication. The interpretation and reporting of these data are the sole responsibility of the authors, and no endorsement by the Norwegian Patient Registry is intended nor should be inferred.

Contributors: BP, PU, and HS initiated the study. BP and HS had full access to all the data in the study and take responsibility for the integrity of the data and the accuracy of the data analysis. BP drafted the manuscript. HS performed the statistical analysis. All authors contributed to the design of the study; the acquisition, analysis, and interpretation of data; and to the critical revision of the manuscript for important intellectual content. BP supervised the study and is the guarantor. The corresponding author attests that all listed authors meet authorship criteria and that no others meeting the criteria have been omitted.

Funding: The study was conducted with unrestricted research grant support from the Swedish Heart-Lung Foundation, Novo Nordisk Foundation, and Swedish Society for Medical Research. BP was supported by an investigator grant from the Strategic Research Area 
Epidemiology programme at the Karolinska Institutet. The funding sources had no role in the design and conduct of the study; collection, management, analysis, and interpretation of the data; preparation, review, or approval of the manuscript; and decision to submit the manuscript for publication.

Competing interests: All authors have completed the ICMJE uniform disclosure form at www.icmje.org/coi_disclosure.pdf (available on request from the corresponding author) and declare: support from the Swedish Heart-Lung Foundation, Novo Nordisk Foundation, and Swedish Society for Medical Research for the submitted work; BE has received personal fees for lectures and serving on advisory boards from Amgen, AstraZeneca, Boerhringer Ingelheim, Eli Lilly, Merck Sharp and Dohme, Mundipharma, Navamedic, Novo Nordisk, and RLS Global outside the submitted work, and research grants from Sanofi outside the submitted work; CJ has received personal fees for research consultancy work from Pfizer and Bayer outside of the submitted work; HS has received consulting fees from Celgene and is employed by IQVIA outside of the submitted work; SG has received lecture fees and research grants from AstraZeneca, Boerhringer Ingelheim, Eli Lilly, Merck Sharp and Dohme, Novo Nordisk, and Sanofi outside of the submitted work; the other authors did not have any potential competing interests to report.

Ethical approval: The study was approved by the regional ethical review board in Stockholm, Sweden (registration number 2016/2029. $31 / 1$ ), and the regional committee for medical and health research ethics in Norway (REC Central, registration number 2016/1959). In Denmark, ethical approval is not required for register based research. Data sharing: No additional data available.

The lead author affirms that the manuscript is an honest, accurate, and transparent account of the study being reported; that no important aspects of the study have been omitted; and that any discrepancies from the study as planned have been explained.

This is an Open Access article distributed in accordance with the Creative Commons Attribution Non Commercial (CC BY-NC 4.0) license, which permits others to distribute, remix, adapt, build upon this work non-commercially, and license their derivative works on different terms, provided the original work is properly cited and the use is noncommercial. See: http://creativecommons.org/licenses/by-nc/4.0/.

1 Wiviott SD, Raz I, Bonaca MP, et al, DECLARE-TIMI 58 Investigators. Dapagliflozin and cardiovascular outcomes in type 2 diabetes. $N$ Engl J Med 2019;380:347-57. doi:10.1056/NEJMoa1812389

2 Neal B, Perkovic V, Mahaffey KW, et al, CANVAS Program Collaborative Group. Canagliflozin and cardiovascular and renal events in type 2 diabetes. N Engl J Med 2017;377:644-57. doi:10.1056/ NEJMoa1611925

3 Zinman B, Wanner C, Lachin IM, et al, EMPA-REG OUTCOME Investigators. Empagliflozin, cardiovascular outcomes, and mortality in type 2 diabetes. N Engl J Med 2015;373:2117-28. doi:10.1056/ NEJMoa1504720

4 Zelniker TA, Wiviott SD, Raz I, et al. SGLT2 inhibitors for primary and secondary prevention of cardiovascular and renal outcomes in type 2 diabetes: a systematic review and meta-analysis of cardiovascular outcome trials. Lancet 2019;393:31-9. doi:10.1016/S01406736(18)32590-X

5 Kim YG, Han SJ, Kim DJ, Lee KW, Kim HJ. Association between sodium glucose co-transporter 2 inhibitors and a reduced risk of heart failure in patients with type 2 diabetes mellitus: a real-world nationwide population-based cohort study. Cardiovasc Diabetol 2018;17:91 doi:10.1186/s12933-018-0737-5

6 Ryan PB, Buse JB, Schuemie MJ, et al. Comparative effectiveness of canagliflozin, SGLT2 inhibitors and non-SGLT2 inhibitors on the risk of hospitalization for heart failure and amputation in patients with type 2 diabetes mellitus: A real-world meta-analysis of 4 observational databases (OBSERVE-4D). Diabetes Obes Metab 2018;20:2585-97. doi:10.1111/dom.13424

7 Kosiborod M, Cavender MA, Fu AZ, et al, CVD-REAL Investigators and Study Group*. Lower risk of heart failure and death in patients initiated on sodium-glucose cotransporter-2 inhibitors versus other glucose-lowering drugs: The CVD-REAL study (comparative effectiveness of cardiovascular outcomes in new users of sodiumglucose cotransporter-2 inhibitors). Circulation 2017;136:249-59. doi:10.1161/CIRCULATIONAHA.117.029190

8 Birkeland $\mathrm{KI}$, Jørgensen ME Carstensen $\mathrm{B}$, et al Cardiovascular mortality and morbidity in patients with type 2 diabetes following initiation of sodium-glucose co-transporter-2 inhibitors versus other glucose-lowering drugs (CVD-REAL Nordic): a multinational observational analysis. Lancet Diabetes Endocrinol 2017;5:709-17. doi:10.1016/S2213-8587(17)30258-9

9 Kosiborod M, Lam CSP, Kohsaka S, et al, CVD-REAL Investigators and Study Group. Cardiovascular events associated with SGLT-2 inhibitors versus other glucose-lowering drugs: the CVD-REAL 2 study. J Am Coll Cardiol 2018;71:2628-39. doi:10.1016/j.jacc.2018.03.009

10 Persson F, Nyström T, Jørgensen ME, et al. Dapagliflozin is associated with lower risk of cardiovascular events and all-cause mortality in people with type 2 diabetes (CVD-REAL Nordic) when compared with dipeptidyl peptidase-4 inhibitor therapy: A multinational observational study. Diabetes Obes Metab 2018;20:344-51. doi:10.1111/dom.13077

11 Udell JA, Yuan Z, Rush T, Sicignano NM, Galitz M, Rosenthal N. Cardiovascular outcomes and risks after initiation of a sodium glucose cotransporter 2 inhibitor: results from the EASEL population-based cohort study (evidence for cardiovascular outcomes with sodium glucose cotransporter 2 inhibitors in the real world). Circulation 2018;137:1450-9. doi:10.1161/ CIRCULATIONAHA.117.031227

12 Suissa S. Reduced mortality with sodium-glucose cotransporter-2 inhibitors in observational studies: avoiding immortal time bias. Circulation 2018;137:1432-4. doi:10.1161/ CIRCULATIONAHA.117.032799

13 Suissa S. Lower risk of death with SGLT2 inhibitors in observational studies: real or bias?Diabetes Care 2018;41:6-10. doi:10.2337/ dc17-1223

14 Patorno E, Goldfine AB, Schneeweiss S, et al. Cardiovascula outcomes associated with canagliflozin versus other nongliflozin antidiabetic drugs: population based cohort study. $B M / 2018 ; 360: k 119$. doi:10.1136/bmi.k119

15 Lund JL, Richardson DB, Stürmer T. The active comparator, new user study design in pharmacoepidemiology: historical foundations and contemporary application. Curr Epidemiol Rep 2015;2:221-8. doi:10.1007/s40471-015-0053-5

16 Ueda P, Svanström H, Melbye M, et al. Sodium glucose cotransporter 2 inhibitors and risk of serious adverse events: nationwide register based cohort study. BMI 2018:363:k4365. doi:10.1136/bmj.k4365

17 Svanström H, Ueda P, Melbye M, et al. Use of liraglutide and risk of major cardiovascular events: a register-based cohort study in Denmark and Sweden. Lancet Diabetes Endocrinol 2019;7:106-14. doi:10.1016/S2213-8587(18)30320-6

18 D’Agostino RBJr, Rubin DB. Estimating and using propensity scores with partially missing data. J Am Stat Assoc 2000;95:749-59. doi:10. 1080/01621459.2000.10474263

19 Ludvigsson JF, Andersson E, Ekbom A, et al. External review and validation of the Swedish national inpatient register. BMC Public Health 2011:11:450. doi:10.1186/1471-2458-11-450

20 Schmidt M, Schmidt SA, Sandegaard JL, Ehrenstein V, Pedersen L, Sørensen HT. The Danish National Patient Registry: a review of content, data quality, and research potential. Clin Epidemiol 2015;7:449-90. doi:10.2147/CLEP.S91125

21 Sundbøll J, Adelborg K, Munch T, et al. Positive predictive value of cardiovascular diagnoses in the Danish National Patient Registry: a validation study. BMJ Open 2016;6:e012832. doi:10.1136/ bmjopen-2016-012832

22 Fralick M, Schneeweiss S, Patorno E. Risk of Diabetic Ketoacidosis after Initiation of an SGLT2 Inhibitor. N Engl J Med 2017;376:2300-2. doi:10.1056/NEJMc1701990

23 Blau JE, Tella SH, Taylor SI, Rother KI. Ketoacidosis associated with SGLT2 inhibitor treatment: Analysis of FAERS data. Diabetes Metab Res Rev 2017;33. doi:10.1002/dmrr.2924

24 Chang HY, Singh S, Mansour O, Baksh S, Alexander GC. Association between sodium-glucose cotransporter 2 inhibitors and lower extremity amputation among patients with type 2 diabetes. JAMA Intern Med 2018;178:1190-8. doi:10.1001/ jamainternmed.2018.3034

25 Harel O, Zhou XH. Multiple imputation: review of theory, implementation and software. Stat Med 2007;26:3057-77. doi:10.1002/sim.2787

26 Patorno E, Pawar A, Franklin JM, et al. Empagliflozin and the risk of heart failure hospitalization in routine clinical care. Circulation 2019;139:2822-30. doi:10.1161/CIRCULATIONAHA.118.039177

27 Zhang XL, Zhu QQ, Chen YH, et al. Cardiovascular safety, longterm noncardiovascular safety, and efficacy of sodium-glucose cotransporter 2 inhibitors in patients with type 2 diabetes mellitus: a systemic review and meta-analysis with trial sequential analysis. / Am Heart Assoc 2018:7:e007165 doi:10.1161/JAHA.117.007165

28 Yang JY, Wang T, Pate V, et al. Sodium-glucose co-transporter-2 inhibitor use and risk of lower-extremity amputation: Evolving questions, evolving answers. Diabetes Obes Metab 2019;21:122336. doi:10.1111/dom.13647

29 Perkovic V, Jardine MJ, Neal B, et al, CREDENCE Trial Investigators. Canagliflozin and renal outcomes in type 2 diabetes and nephropathy. N Engl J Med 2019;380:2295-306. doi:10.1056/ NEJMoa1811744

30 Scirica BM, Bhatt DL, Braunwald E, et al, SAVOR-TIMI 53 Steering Committee and Investigators. Saxagliptin and cardiovascular outcomes in patients with type 2 diabetes mellitus. N Engl f Med 2013;369:1317-26. doi:10.1056/NEJMoa1307684 
31 Green JB, Bethel MA, Armstrong PW, et al, TECOS Study Group. Effect of sitagliptin on cardiovascular outcomes in type 2 diabetes. $N$ Engl Med 2015:373:232-42. doi:10.1056/NEIMoa1501352

32 Zannad F, Cannon CP, Cushman WC, et al, EXAMINE Investigators. Heart failure and mortality outcomes in patients with type 2 diabetes taking alogliptin versus placebo in EXAMINE: a multicentre, randomised, double-blind trial. Lancet 2015;385:2067-76. doi:10.1016/S0140-6736(14)62225-X

33 Rosenstock J, Perkovic V, Johansen OE, et al, CARMELINA Investigators. Effect of linagliptin vs placebo on major cardiovascular events in adults with type 2 diabetes and high cardiovascular and renal risk: the CARMELINA randomized clinical trial. JAMA 2019;321:69-79. doi:10.1001/jama.2018.18269

34 Filion KB, Azoulay L, Platt RW, et al, CNODES Investigators. A multicenter observational study of incretin-based drugs and heart failure. N Engl/ Med 2016;374:1145-54. doi:10.1056/NEJMoa1506115

35 Toh S, Hampp C, Reichman ME, et al. Risk for hospitalized heart failure among new users of saxagliptin, sitagliptin, and other antihyperglycemic drugs: a retrospective cohort study. Ann Intern

Web appendix: Supplementary appendix 